\title{
Epitaxial Growth of Germanium on Silicon for Light Emitters
}

\author{
Chengzhao Chen, ${ }^{1,2}$ Cheng Li, ${ }^{1}$ Shihao Huang, ${ }^{1}$ Yuanyu Zheng, ${ }^{1}$ \\ Hongkai Lai, ${ }^{1}$ and Songyan Chen ${ }^{1}$ \\ ${ }^{1}$ Department of Physics, Semiconductor Photonics Research Center, Xiamen University, Xiamen, Fujian 361005, China \\ ${ }^{2}$ Department of Physics and Electronic Engineering, Hanshan Normal University, Chaozhou 521041, China
}

Correspondence should be addressed to Cheng Li, lich@xmu.edu.cn

Received 30 June 2011; Revised 20 August 2011; Accepted 24 August 2011

Academic Editor: Fabrice Gourbilleau

Copyright ( $) 2012$ Chengzhao Chen et al. This is an open access article distributed under the Creative Commons Attribution License, which permits unrestricted use, distribution, and reproduction in any medium, provided the original work is properly cited.

\begin{abstract}
This paper describes the role of Ge as an enabler for light emitters on a Si platform. In spite of the large lattice mismatch of $\sim 4.2 \%$ between $\mathrm{Ge}$ and $\mathrm{Si}$, high-quality Ge layers can be epitaxially grown on Si by ultrahigh-vacuum chemical vapor deposition. Applications of the Ge layers to near-infrared light emitters with various structures are reviewed, including the tensile-strained Ge epilayer, the Ge epilayer with a delta-doping SiGe layer, and the Ge/SiGe multiple quantum wells on Si. The fundamentals of photoluminescence physics in the different Ge structures are discussed briefly.
\end{abstract}

\section{Introduction}

In the past decades, the zeal for investigating germanium has been stimulated by its novel application in electronic and optoelectronic devices. Due to its superior electron and hole mobility compared to $\mathrm{Si}$, Ge has emerged as a feasible candidate to maintain performance for future electronic applications [1]. In addition, the small direct energy band gap of $0.8 \mathrm{eV}$ at room temperature in $\mathrm{Ge}$ makes it possible to design efficient high speed $(\geq 40 \mathrm{GHz})$ reliable photodetectors operating in the low-loss optical fiber range of $1.3 \sim 1.5 \mu \mathrm{m}[2-4]$. Ge layers can also be used in conjunction with advanced gate dielectrics such as $\mathrm{HfO}_{2}$ for the formation of bulk Ge [5, 6] or Ge-on-insulator- (GeOI-) [79] based metal oxide semiconductor field effect transistors (MOSFETs) with superior hole mobility. Ge has a relatively small energy difference of $0.14 \mathrm{eV}$ between the indirect $L$ valley and the direct $\Gamma$ valley. This difference is reduced to $\sim 0.11 \mathrm{eV}$ under the tensile strain as large as $0.2 \%$ [10]. In heavily doped $\mathrm{n}^{+}$-Ge, the electron scattering between the $\Gamma$ and $L$ valleys could be slowed down, showing a bright photoluminescence $(\mathrm{PL})$ in the $1.55 \mu \mathrm{m}$ range $[11,12]$ and attempting to achieve an optical gain due to the direct transition in $\mathrm{Ge}[13,14]$. More recently, light-emitting diodes using $\mathrm{Ge}$ pin structures on $\mathrm{Si}$ have been demonstrated and the first optically pumped Ge-on-Si laser operating at room temperature was fabricated $[15,16]$. Finally, due to their small lattice mismatch with GaAs $\left(a_{\mathrm{Ge}}=0.565785 \mathrm{~nm}\right.$, $a_{\mathrm{GaAs}}=0.56533 \mathrm{~nm}$ ) and similar thermal expansion coefficients, Ge layers can be used as templates for the growth of GaAs-based heterostructures such as diodes and solar cells [17], laser diodes [18], high electron mobility and heterojunction bipolar transistors $[19,20]$.

However, the central issue in obtaining useful Ge films on $\mathrm{Si}$ is overcoming the negative effects of the difference in thermal expansion coefficient and the large lattice mismatch $(4.2 \%$ at $300 \mathrm{~K})$ between these two materials, which cause (i) a high density of misfit dislocations at the interface and a high threading dislocation density (TDD) in the Ge layers, which severely affects the performance of Ge devices because of the recombination centres that are introduced along these dislocations, and (ii) high surface roughness due to island growth, making subsequent device processing very difficult because complementary metal-oxide-semiconductor (CMOS) devices require planar processing. Many recent studies have explored the deposition of Ge films on Si. For example, a method using graded SiGe buffer layers reduced the TDD to the range of $10^{6} \sim 10^{7} \mathrm{~cm}^{-2}$ [21]. However, a $10 \mathrm{~mm}$-thick graded layer was required to achieve the $10^{6} \mathrm{~cm}^{-2}$ defects level, and this had to be followed by chemical mechanical polishing to obtain a smooth surface, which is not appropriate for monolithic integration of devices on 
Si and has poor thermal conduction. An advantage of the low-energy plasma-enhanced chemical vapour deposition (LEPECVD) technique is the high growth rate achievable on the order of $4 \mathrm{~nm} /$ second, allowing thick SiGe-graded buffer layers to be grown faster than by other epitaxial techniques and thereby increasing throughput in order to make such structures more manufacturable, and therefore relaxed $\mathrm{Ge}$ on a silicon substrate with a threading dislocation density of $1 \times 10^{5} \mathrm{~cm}^{-2}$ was achieved [22]. Direct pure Ge deposition on $\mathrm{Si}$ has been done with cyclic thermal annealing with a reduced pressure CVD system, which reduces the TDD $\left(6 \times 10^{6} \mathrm{~cm}^{-2}\right.$ with $2.5 \mathrm{~mm}$-thick Ge layer $)$ and also creates rather smooth surface roughness (root-mean-square (RMS) surface roughness: $\sim 1 \mathrm{~nm})[2,23]$. Nayfeh et al. showed that high-temperature (HT) hydrogen annealing following low-temperature (LT) deposition $\left(400^{\circ} \mathrm{C}\right)$ reduced the surface RMS roughness from 24 to $2.9 \mathrm{~nm}$ with a $155 \mathrm{~nm}$-thick sample [24]. Liu et al. [25] proposed a defectnecking technique, which confines the defects to short distances by patterning Ge films followed by a conformal low-temperature oxide (LTO) deposition by low pressure chemical vapor deposition (LPCVD) at $300^{\circ} \mathrm{C}$. Vanamu et al. [26] have reported that high-quality Ge layers, with dislocation density consistently lower than $5 \times 10^{5} \mathrm{~cm}^{-2}$, grew on nanostructured Si substrates, which were fabricated by using interferometric lithography combined with reactive ion etching and thermal oxidation methods. Most results have, however, shown that obtaining low TDD and a smooth surface layer at the same time is still very problematic.

\section{Epitaxial Growth of Ge on Si with Low Dislocation Density}

High-quality epitaxial growth of Ge on Si has been realized by using an ultrahigh-vacuum chemical vapor deposition (UHV/CVD) technique. Langdo et al. [27] showed that pure Ge grown selectively on $\mathrm{SiO}_{2} / \mathrm{Si}$ substrates in $100 \mathrm{~nm}$ holes is highly perfect at the top surface compared to conventional Ge lattice-mismatched growth on planar $\mathrm{Si}$ substrates. This result is achieved through a combination of interferometric lithography $\mathrm{SiO}_{2} / \mathrm{Si}$ substrate patterning and ultrahigh vacuum chemical vapor deposition Ge selective epitaxial growth. This "epitaxial necking," in which threading dislocations are blocked at oxide sidewalls, shows promise for dislocation filtering and the fabrication of low-defect density Ge on Si. Defects at the Ge film surface only arise at the merging of epitaxial lateral overgrowth fronts from neighboring holes. Luan et al. [28] reported that the smooth Ge layer on $\mathrm{Si}$ is available using a UHV/CVD growth with a low-high temperature two-step growth technique, which is now employed in the worldwide. In this technique, contrary to the thick SiGe-graded buffer approach, a pure Ge heterolayer as thin as $30 \mathrm{~nm}$, is deposited directly on Si at a low temperature of $300 \sim 400^{\circ} \mathrm{C}$, followed by a highertemperature growth (typically $600^{\circ} \mathrm{C}$ ) with a larger growth rate. The low-temperature Ge buffer layer prevents the threedimensional nucleation of Ge. Recently, Loh et al. [29] reported a modified two-step growth approach, that is, growing an ultrathin $(2 \sim 30 \mathrm{~nm}) \mathrm{LT} \mathrm{SiGe} \mathrm{buffer} \mathrm{layer} \mathrm{prior} \mathrm{to}$

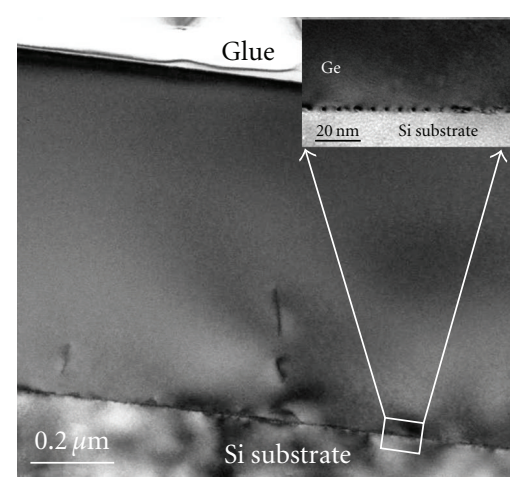

(a)

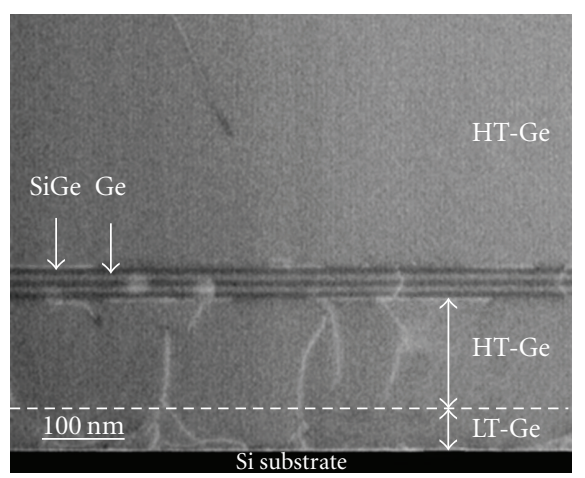

(b)

Figure 1: Transmission electron microscope cross-sections of Geon-Si layers for (a) typical and (b) modified two-step methods.

the deposition of LT Ge seed layer and HT Ge layer. With the help of LT SiGe layer to absorb partially misfit strain, provide Ge nucleation sites, and coalesce dislocations, the TDD can be reduced to $6 \times 10^{6} \mathrm{~cm}^{-2}$, without any thermal annealing.

Recently, we investigate the growth of the Ge epilayer on Si by UHV/CVD combined with the advantages of the low-temperature buffer layer and strained layer superlattices (SLSs). In the initial growth step, a thin epitaxial Ge buffer layer of $80 \mathrm{~nm}$ was directly grown on $\mathrm{Si}$ at $350^{\circ} \mathrm{C}$. After that, $220 \mathrm{~nm} \mathrm{HT}$ Ge layer was grown at $630^{\circ} \mathrm{C}$ and then 3-period $\mathrm{SiGe} / \mathrm{Ge}$-strained layer superlattices (SLSs) was introduced as an intermediate layer for further improving the quality of the top Ge layer. Finally, the growth temperature in the main growth step was increased to $630^{\circ} \mathrm{C}$ to achieve higher growth rates and better crystal quality. The high-quality Ge epilayer on Si was achieved with a surface RMS roughness of less than $1 \mathrm{~nm}$ and a TDD of $1.5 \times 10^{6} \mathrm{~cm}^{-2}$.

Figure 1 shows cross-sectional transmission electron microscope images of Ge-on-Si by the typical and our modified two-step methods. It can be seen in Figure 1(a) that most of misfit dislocations are confined at the LT$\mathrm{Ge}$ and $\mathrm{Si}$ interface as shown in the inset. Some of them thread upward and generate threading dislocations at the surface and then a number of them meet and annihilate in the region of HT-Ge layer close to the LT-Ge interface. Figure 1(b) clearly shows that the dislocations start at the bottom interface and propagate toward the upper layer till 


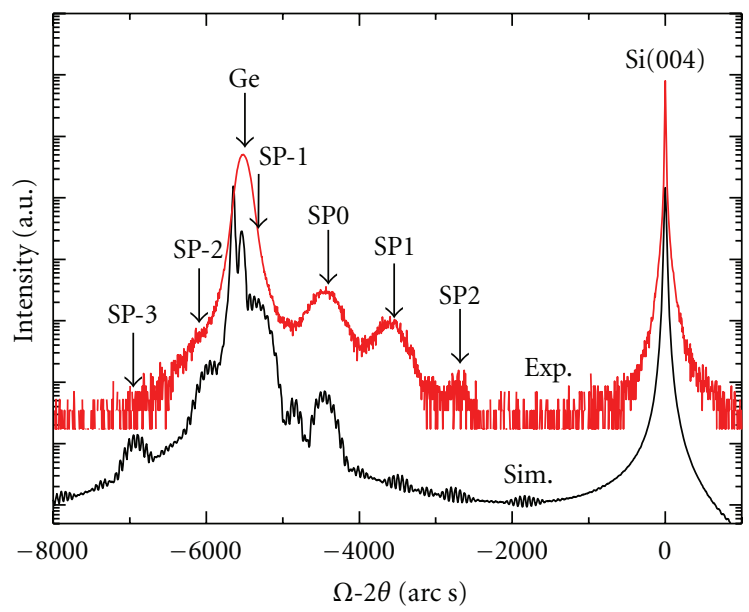

FIGURE 2: XRD $\Omega-2 \theta$ profiles of Ge epilayer on LT-Ge layer ans SLSs.

the annihilation reactions occurred, and there is almost no dislocation threading from the first HT-Ge layer to the HT-Ge epilayer. The observation indicated a possible way for dislocation reduction: the separated threading dislocations are driven by the force as a result of the strain accumulated in the SiGe/Ge SLSs and coalesces or annihilation in the end. The TEM observations in Figure 1 confirm that a further reduction of threading dislocations can be achieved by combining with the advantages of low-temperature buffer layer and strained layer superlattices.

The strain status and crystal quality of Ge layers were evaluated by double crystal XRD measurement (Bede, D1 system), using a $\mathrm{Cu} \mathrm{K}_{\mathrm{a} 1}(\lambda=0.15406 \mathrm{~nm})$ as the $\mathrm{X}$-ray source. The $\Omega-2 \theta$ symmetric (lllll $\left.\begin{array}{lll}0 & 0 & 4\end{array}\right)$ XRD scan of Ge epilayer is shown in Figure 2, including the result of the simulation of the XRD patterns based on the dynamical diffraction theory. Besides the peak originating from the Ge layer, multiple high order superlattice satellites are also clearly observed for the sample, suggesting the interface between Ge layers and SiGe layers sharp and clear. The peak distance between Ge epilayer and $\mathrm{Si}$ is 5525. This value is smaller than the expected one between the fully relaxed Ge and Si. According to Bragg's law with $\lambda=0.15406 \mathrm{~nm}$ for $\mathrm{CuK}_{a 1}$ radiation, the peak distance is 5649 arc sec with the relaxed lattice constants 0.5431 and $0.5658 \mathrm{~nm}$ for $\mathrm{Si}$ and Ge. The smaller peak distance between epitaxial Ge and Si substrate indicates that the epitaxial Ge layer is under tensile strain [30]. From the peak positions, the in-plane lattice constant is evaluated to be $0.5674 \mathrm{~nm}$ for the sample, corresponding to the in-plane tensile strain of $0.13 \%$. The strain in the Ge epilayer mainly arises from the differences in the thermal expansion coefficients between $\mathrm{Ge}$ and $\mathrm{Si}$ and accumulates in the layers during cooling process from elevated growth temperature to room temperature. Figure 3 shows the atomic force microscope(AFM) image of the sample. The surface is found to consist of step and terrace structures, indicating the two-dimensional layer-tolayer growth. A small RMS surface roughness of $0.45 \mathrm{~nm}$ is obtained.

Figures 4(a) and 4(b) show depth profile of TDD in the Ge epilayer on Si substrate and several typical optical

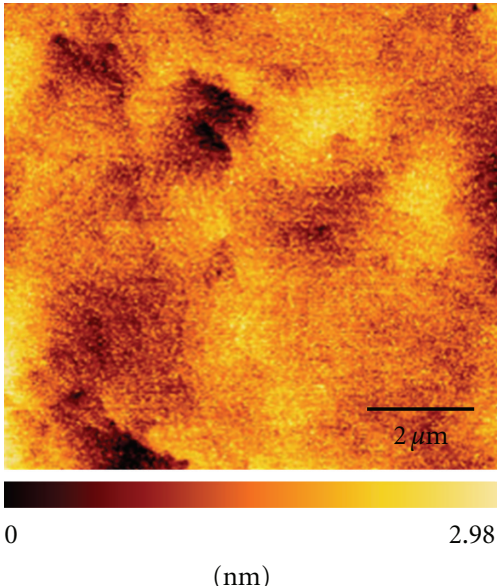

Figure 3: A typical AFM image for Ge surface.

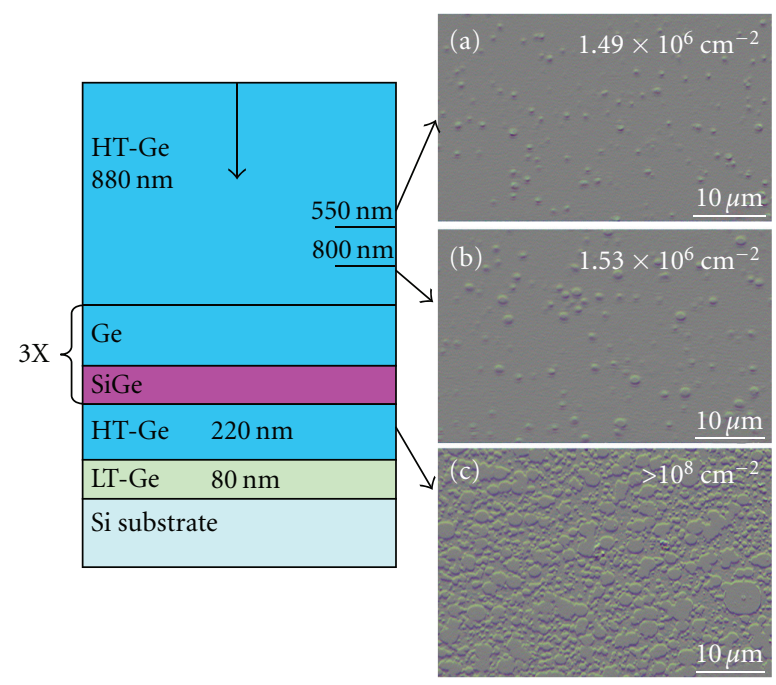

FIGURE 4: Schematic and optical images of the sample etched at various depths to reveal etch pit density.

images at various depths in the sample after etched by $\mathrm{I}_{2}$ solution (HF: $\mathrm{HNO}_{3}: \mathrm{CH}_{3} \mathrm{COOH}: \mathrm{I}_{2}=10 \mathrm{~mL}: 40 \mathrm{~mL}$ : $100 \mathrm{~mL}: 30 \mathrm{mg}$ ) for EPD counting. It is found that TDDs weakly depend on the etch depth when the retained $\mathrm{Ge}$ layer is larger than $80 \mathrm{~nm}$ from SLSs and only the size of the etch pits become larger with increasing etch depth. The typical TDD is in a range of $1.49 \times 10^{6} \mathrm{~cm}^{-2} \sim 1.53 \times$ $10^{6} \mathrm{~cm}^{-2}$. When the Ge layer is etched to be left less than $270 \mathrm{~nm}$ from the Si substrate, TDDs in the first HT-Ge layer increase quickly to larger than $1 \times 10^{8} \mathrm{~cm}^{-2}$ (limitation of EPD method), and the surface morphology image shown as Figure 4(c). The results indicate that the SLSs can partly filter the thread dislocations, in accordance with the TEM analyses.

\section{Ge on Si for Light Emitters}

The realization of silicon photonics requires a Si-based light emitter capable of integration with electronic integrated circuits. Ge has been proposed as a very promising candidate 


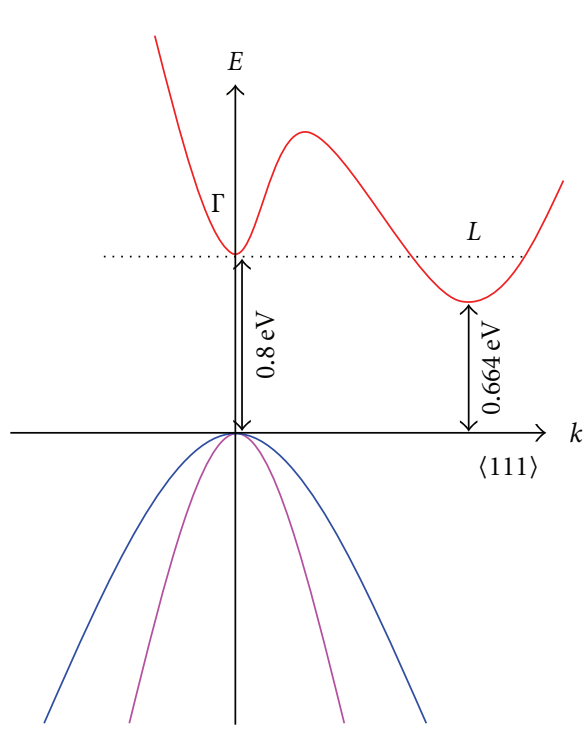

(a)

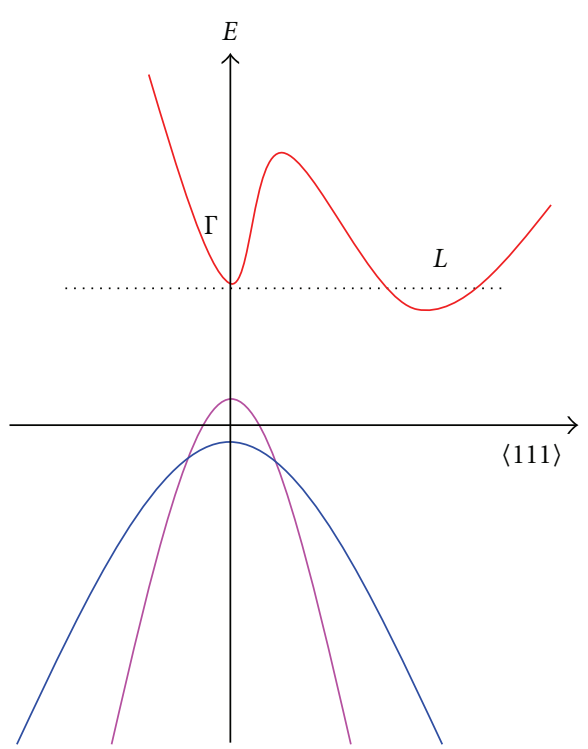

(b)

FIGURE 5: (a) Schematic band structure of bulk, showing a $136 \mathrm{meV}$ difference between the direct and indirect gap, (b) the difference between the direct and indirect gap can be decrease by tensile strain.

to make such a light emitter for $\mathrm{Ge}$ is a $\mathrm{Si}$ compatible material and a pseudodirect gap behavior because the energy difference between its direct and indirect bandgaps is only $136 \mathrm{meV}$ at room temperature. Ge is normally recognized as a poor light-emitting material due to its indirect band structure. The radiative recombination through indirect transition is inefficient as a result of a phonon-assisted process. Therefore, indirect gap PL was only observed from highpurity single crystalline bulk $\mathrm{Ge}$ at cryogenic temperatures. The direct transition in $\mathrm{Ge}$, on the other hand, is a very fast process with radiative recombination rate of four and five orders of magnitude higher than that of the indirect transition, so that the direct gap light emission of Ge is as efficient as that of direct gap III-V materials. The challenge is to have a sufficient number of electrons in the direct valley of the conduction band because most of the electrons are pumped into the lower energy indirect $L$ valleys (fourfold degenerate) following the Fermi distribution.

To turn Ge into an efficient light-emitting material, we have to compensate for the difference between the direct and indirect bandgaps. It has been demonstrated that this difference can be decreased by introducing tensile strain into the Ge layer, and it has been applied to improve the performance of Ge light emitting on Si. Another strategy is to compensate for the rest of the energy difference by n-type doping to fill electrons into the $L$ valleys up to the level of the $\Gamma$ valley. With these methods, the tensile-strained n-type Ge effectively provides for population inversion in the direct bandgap, leading to strong light emission from its direct bandgap transitions.

The band structure of bulk Ge is schematically shown in Figure 5(a), with a $0.664 \mathrm{eV}$ indirect band gap at the $L$ valleys and a $0.800 \mathrm{eV}$ direct bandgap at the $\Gamma$ valley. Under the tensile strain, the direct bandgap energies of Ge are reduced.

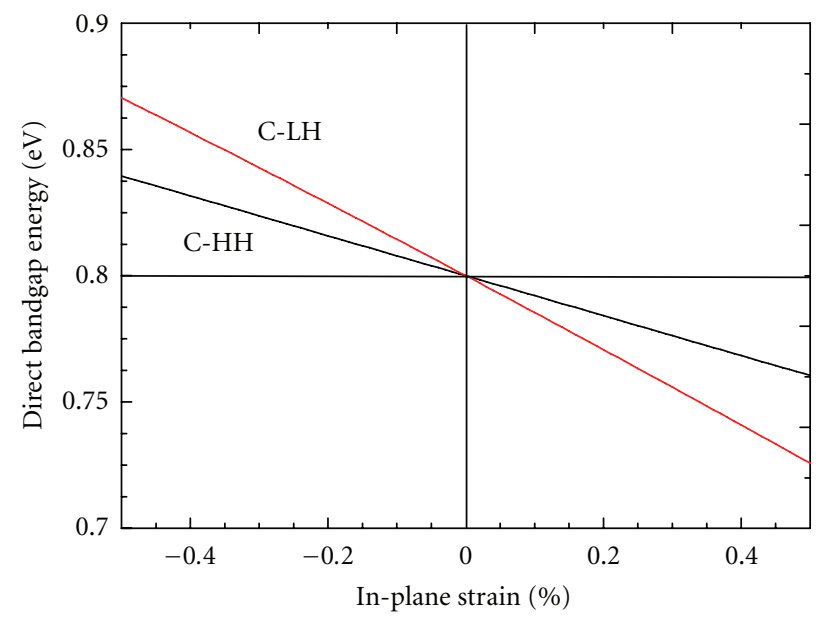

FIGURE 6: Direct bandgap energies of $\mathrm{Ge}$ as a function of in-plane biaxial strain.

Figure 6 shows a calculation result based on the deformation potential theory [31]. Photoluminescence of the samples of tensile strained Ge grown on Si substrate, as well as bulk Ge is shown in Figure 7. It is observed that PL main peak of the tensile-strained Ge shifts to the low energy comparing to that of the bulk Ge [32]. This result demonstrates that the tensilestrain in the Ge epitaxial layer induces the reduction in direct band gap.

An enhancement of the direct bandgap photoluminescence from Ge layer on silicon with boron or phosphorous delta-doping SiGe layers at room temperature is reported [33]. The n-type delta-doping SiGe layer is proposed to transfer extra electrons to $L$ valley in Ge, which decreases the possibility of the excited electrons in the delta valley to be scattered to the $L$ valley and improves the photoluminescence 


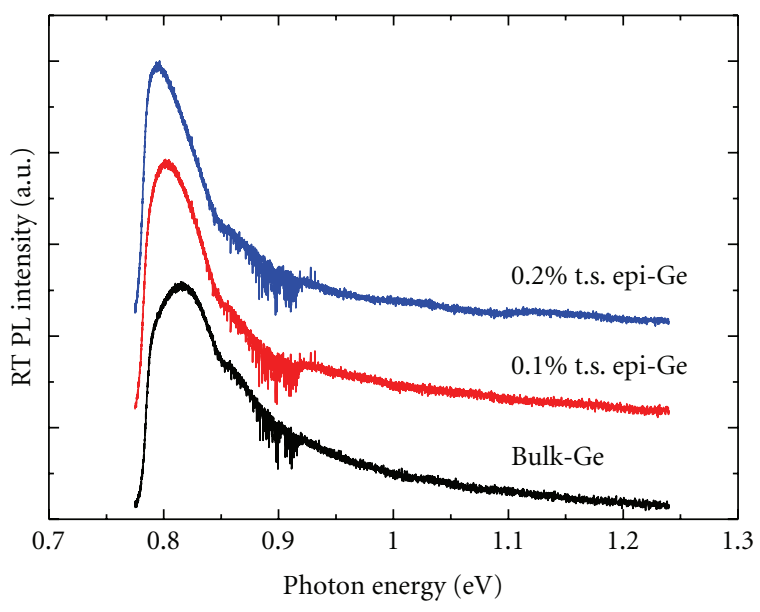

FIGURE 7: Room temperature photoluminescence of tensilestrained Ge on Si.

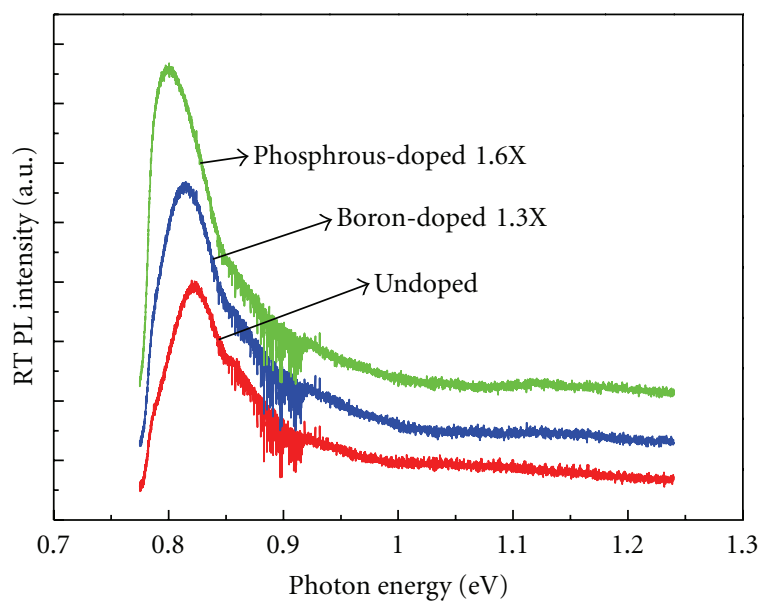

FIGURE 8: Room temperature photoluminescence of tensilestrained $\mathrm{Ge}$ on Si with delta-doping SiGe layers.

of the direct band transition in the Ge layer. While precluding the introduction of additional nonradiative recombination centers in the Ge layer.

Room temperature PL spectra of the samples with p- or n-delta-doping SiGe are shown in Figure 8. The PL signal is enhanced by a factor of 1.3 for the sample with the ptype delta-doping SiGe layer and 1.6 for the sample with the n-type doping SiGe layer compared to that of the sample with the undoped SiGe layer. The shape and full width at half maximum (FWHM) of the luminescence spectra of the samples with the doped or undoped SiGe layer is almost the same, suggesting that the luminescence of all the samples should be ascribed to the same origin. In order to well understand the enhancement of luminescence from Ge with the delta-doping SiGe layers, the bandgap structure was calculated by effective mass approximation method including the strain affects in Ge and SiGe layers. Because of the requirement of the same Fermi levels in equilibrium, the activated holes in the boron-doped SiGe layer will transfer to the Ge layer and occupy the top of the valence band, which will induce the band bending near the interface

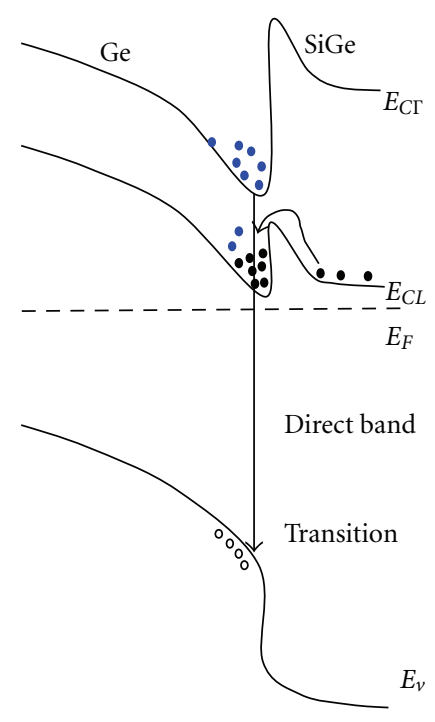

- Excited

- Preoccupation

FIGURE 9: The schematic of band gap of Ge/phosphorous-doped SiGe structure.

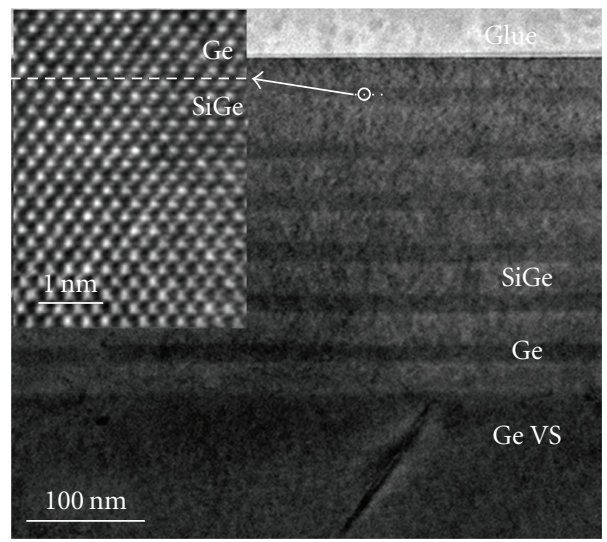

FIgure 10: HRTEM images of sample with Ge/SiGe MQW on Geon-Si substrate.

and increase the possibility of the direct band transitions of the laser-excited electrons in the $\Gamma$ valley due to the coulomb interaction. This results in a little bit enhancement of luminescence. For the sample with the phosphorous deltadoping SiGe layer, the Fermi level is under the indirect conduction band ( $L$ valley) with the doping concentration of $5 \times 10^{17} \mathrm{~cm}^{-3}$ in the SiGe layer, the electrons in the SiGe layer will transfer and then be confined in the Ge well by the potential barrier of several tens $\mathrm{meV}$ due to the energy band shift of the Ge/SiGe heterostructure. The confined electrons will naturally occupy the $L$ valley in the Ge layer. The electron preoccupation of the $L$ valley will significantly decrease the possibility of the excited electrons in the $\Gamma$ valley to be scattered to the $L$ valley and increase the possibility of direct band transitions, as schematically described in Figure 9. This will result in more effective enhancement of luminescence 


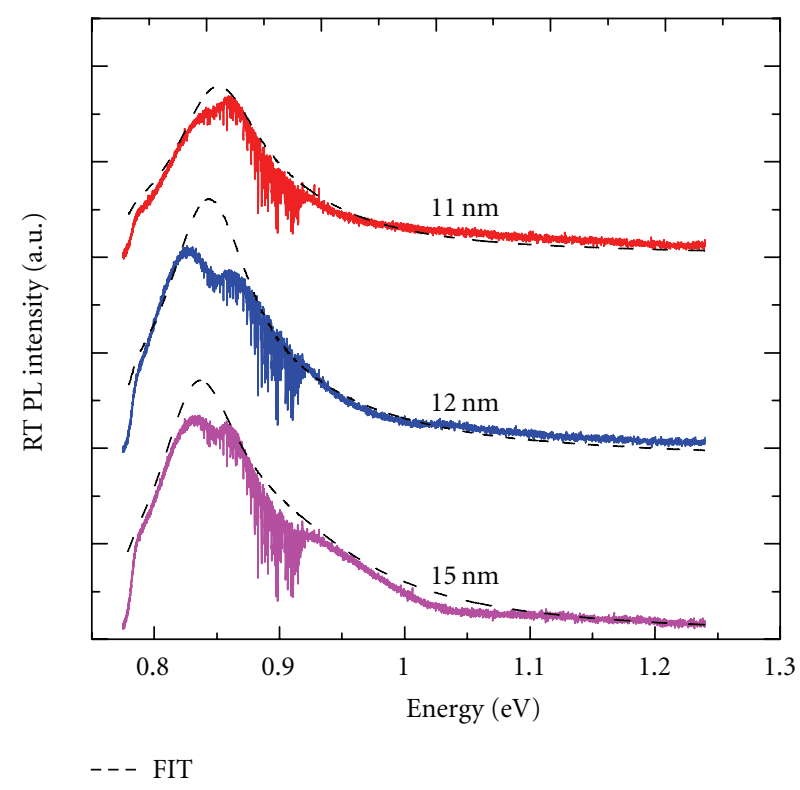

(a)

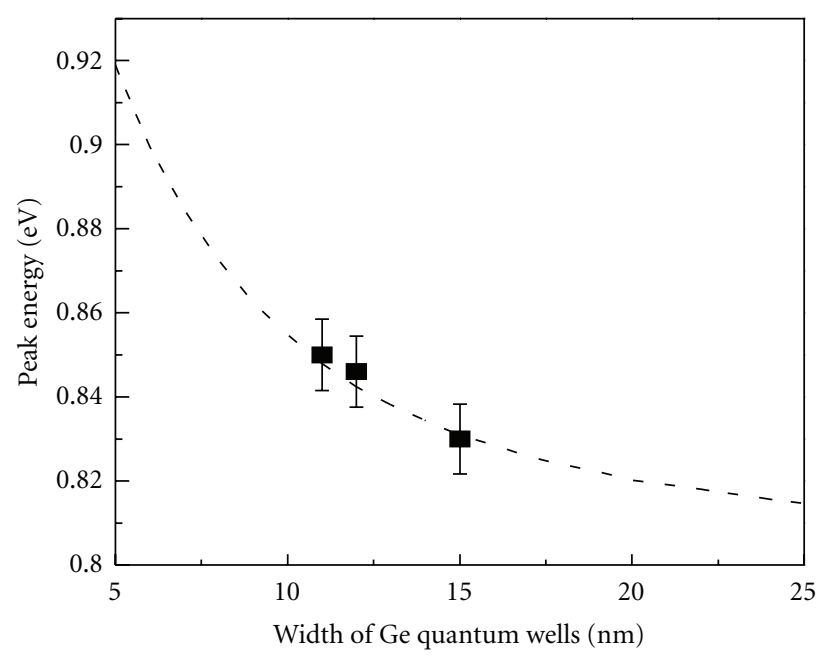

(b)

FIGURE 11: (a) Room temperature photoluminescence of the tensile-strained Ge/SiGe MQW of the samples; (b) dependence of photoluminescence peak energy on the thickness of Ge well layer. The calculated direct band transition energy due to quantum confinement effect at $\Gamma$ point is plotted as dashed line.

from Ge layer with the n-type delta-doping SiGe layer than that with the p-type delta-doping or undoped SiGe layers. Inserting an n-type delta-doping SiGe layer is different from the directly doped $\mathrm{Ge}$ and also effective to provide electrons to the Ge layer and exclude the introduction of extranonradiative centers resulting in the improvement of $\mathrm{PL}$ from Ge.

We directly demonstrate quantum-confined direct band transitions in the tensile strained Ge/SiGe multiple quantum wells grown on silicon substrates by room temperature photoluminescence [34]. It is indicated that the photoluminescence peak energy of the tensile-strained Ge/SiGe quantum wells shifts to higher energy with the reduction of thickness of Ge well layers. This blue shift of the luminescence peak energy can be quantitatively explained by the direct band transitions due to the quantum confinement effect at $\Gamma$ point of conduction band.

Figure 10 depicts the cross sectional HRTEM images of the sample with 6 periods of Ge/SiGe MQW on Ge-onSi substrate. It indicates that the surface is smooth and few of dislocations in the Ge/SiGe MQW are observed in the TEM images. The perfect lattice match at the Ge/SiGe interface as shown in the inset suggests that the alternating growth of SiGe and Ge layers is pseudomorphic. Room temperature photoluminescence of the samples with tensilestrained Ge/SiGe MQW on silicon substrates was shown in Figure 11(a). The spectra of the direct band luminescence from all of the samples show broad bands with the distorted shape in the range of $0.85 \mathrm{eV} \sim 0.92 \mathrm{eV}$. The distortion of the photoluminescence spectra can be attributed to the atmospheric water absorption, as reported in [35]. To eliminate the effect of the atmospheric water absorption on the determination of the peak energy, we fit the direct band photoluminescence spectra following the processes described in [35] and shown in Figure 11(a), as dashed lines. It is clearly shown that the peak energy decreases with the increase of the thickness of Ge quantum well layers. Also shown in Figure 11(b) is the fitted photoluminescence peak energy from the samples, which is in good agreement with the theoretical calculation. Those results demonstrate that the room temperature photoluminescence is originated from the quantum-confined direct band transitions in the tensilestrained Ge wells and the peak energy shift results from the increase of energy separation at $\Gamma$ point in the tensile strained $\mathrm{Ge} / \mathrm{SiGe} \mathrm{MQW}$. It is suggested that the tensile-strained $\mathrm{Ge} / \mathrm{SiGe} \mathrm{MQW}$ on Si substrate is one of the promising materials for Si-based integrated photonic devices.

\section{Conclusion}

The possible applications of Ge on Si to active photonic devices of light emitters were reviewed in addition to the high-quality growth of Ge on Si. The high-quality Ge epilayer on $\mathrm{Si}$ was achieved with a surface RMS roughness of less than $1 \mathrm{~nm}$ and a TDD of $1.5 \times 10^{6} \mathrm{~cm}^{-2}$. Room temperature photoluminescence spectra due to direct band transitions in the tensile-strained Ge epilayer, the Ge eiplayer with a deltadoping SiGe layer and the Ge/SiGe multiple quantum wells on $\mathrm{Si}$ are observed. Those results suggest that Ge will play a significant role as an enabler for intergrating active photonic device on $\mathrm{Si}$.

\section{Acknowledgments}

This paper was supported by the National Basic Research Program of China (Grant no. 2007CB613404), the National Natural Science Foundation of China (Grant nos. 61036003, 
60837001, and 61176092), the Fundamental Research Funds for the Central Universities (Grant no. 2010121056), and the National Basic Research Program of China (Grant no. 2012CB933503).

\section{References}

[1] M. L. Lee, E. A. Fitzgerald, M. T. Bulsara, M. T. Currie, and A. Lochtefeld, "Strained Si, SiGe, and Ge channels for highmobility metal-oxide- semiconductor field-effect transistors," Journal of Applied Physics, vol. 97, no. 1, Article ID 011101, 28 pages, 2005.

[2] J. M. Hartmann, A. Abbadie, A. M. Papon et al., "Reduced pressure-chemical vapor deposition of Ge thick layers on $\mathrm{Si}(001)$ for $1.3-1.55-\mu \mathrm{m}$ photodetection," Journal of Applied Physics, vol. 95, no. 10, pp. 5905-5913, 2004.

[3] M. Rouvìre, L. Vivien, X. Le Roux et al., "Ultrahigh speed germanium-on-silicon-on-insulator photodetectors for 1.31 and $1.55 \mu \mathrm{m}$ operation," Applied Physics Letters, vol. 87, no. 23, Article ID 231109, 3 pages, 2005.

[4] L. Vivien, M. Rouvière, J.-M. Fédéli et al., "High speed and high responsivity germanium photodetector integrated in a silicon-on-insulator microwaveguide," Optics Express, vol. 15, no. 15, pp. 9843-9848, 2007.

[5] C. O. Chui, S. Ramanathan, B. B. Triplett, P. C. Mcintyre, and K. C. Saraswat, "Germanium MOS capacitors incorporating ultrathin high-K gate dielectric," IEEE Electron Device Letters, vol. 23, no. 8, pp. 473-475, 2002.

[6] B. Kaczer, J. Francoa, J. Mitarda, P. J. Roussela, A. Velosoa, and G. Groeseneken, "Improvement in NBTI reliability of Si-passivated Ge/high-k/metal-gate pFETs," Microelectronic Engineering, vol. 86, no. 7-9, pp. 1582-1584, 2009.

[7] L. Claveliera, C. Degueta, C. Le Royera, B. Vincenta, J.-F. Damlencourta, and J.-M. Hartmann, "Review of some critical aspects of Ge and GeOI substrates," ECS Transactions, vol. 3, no. 7, pp. 789-805, 2006.

[8] C. Le Royer, L. Clavelier, C. Tabone et al., "105 nm Gate length pMOSFETs with high-K and metal gate fabricated in a Si process line on $200 \mathrm{~mm}$ GeOI wafers," Solid-State Electronics, vol. 52, no. 9, pp. 1285-1290, 2008.

[9] B. Grandchamp, M.-A. Jaud, P. Scheiblin et al., "In-depth physical investigation of GeOI pMOSFET by TCAD calibrated simulation," Solid-State Electronics, no. 1, pp. 67-72, 2011.

[10] J. F. Liu, X. Sun, D. Pan et al., "Tensile-strained, n-type Ge as a gain medium for monolithic laser integration on Si," Optics Express, vol. 15, no. 18, pp. 11272-11277, 2007.

[11] M. El Kurdi, T. Kociniewski, T.-P. Ngo et al., "Enhanced photoluminescence of heavily n-doped germanium," Applied Physics Letters, vol. 94, no. 19, Article ID 191107, 2009.

[12] X. Sun, J. F. Liu, L. C. Kimerling, and J. Michel, "Direct gap photoluminescence of n-type tensile-strained Ge-on-Si," Applied Physics Letters, vol. 95, no. 1, Article ID 011911, 2009.

[13] X. Sun, J. F. Liu, L. C. Kimerling, and J. Michel, "Optical bleaching of thin film Ge on Si," ECS Transactions, vol. 16, no. 10, pp. 88-889, 2008.

[14] J. F. Liu, X. Sun, L. C. Kimerling, and J. Michel, "Direct-gap optical gain of Ge on Si at room temperature," Optics Letters, vol. 34, no. 11, pp. 1738-1740, 2009.

[15] S.-L. Cheng, J. Lu, G. Shambat et al., "Room temperature $1.6 \mu \mathrm{m}$ electroluminescence from Ge light emitting diode on Si substrate," Optics Express, vol. 17, no. 12, pp. 10019-10024, 2009.
[16] J. F. Liu, X. Sun, R. Camacho-Aguilera, L. C. Kimerling, and J. Michel, "Ge-on-Si laser operating at room temperature," Optics Letters, vol. 35, no. 5, pp. 679-681, 2010.

[17] M. J. Archer, D. C. Law, S. Mesropian et al., "GaInP/GaAs dual junction solar cells on Ge/Si epitaxial templates," Applied Physics Letters, vol. 92, no. 10, Article ID 103503, 2008.

[18] O. Kwon, J. J. Boeckl, M. L. Lee, A. J. Pitera, E. A. Fitzgerald, and S. A. Ringel, "Monolithic integration of AlGaInP laser diodes on SiGe/Si substrates by molecular beam epitaxy," Journal of Applied Physics, vol. 100, no. 1, Article ID 013103, 2006.

[19] D. Lubyshev, J. M. Fastenau, Y. Wu et al., "Molecular beam epitaxy growth of metamorphic high electron mobility transistors and metamorphic heterojunction bipolar transistors on Ge and Ge-on-insulator/Si substrates," Journal of Vacuum Science \& Technology B, vol. 26, no. 3, pp. 1115-1119, 2008.

[20] W. K. Liu, D. Lubyshev, J. M. Fastenau et al., "Monolithic integration of InP-based transistors on $\mathrm{Si}$ substrates using MBE," Journal of Crystal Growth, vol. 311, no. 7, pp. 19791983, 2009.

[21] M. T. Currie, S. B. Samavedam, T. A. Langdo, C. W. Leitz, and E. A. Fitzgerald, "Controlling threading dislocation densities in Ge on Si using graded SiGe layers and chemical-mechanical polishing," Applied Physics Letters, vol. 72, no. 14, pp. 17181720, 1998.

[22] S. G. Thomas, S. Bharatan, R. E. Jones et al., "Structural characterization of thick, high-quality epitaxial Ge on Si substrates grown by low-energy plasma-enhanced chemical vapor deposition," Journal of Electronic Materials, vol. 32, no. 9, pp. 976-980, 2003.

[23] J. M. Hartmann, J. F. Damlencourt, Y. Bogumilowicz, P. Holliger, G. Rolland, and T. Billon, "Reduced pressurechemical vapor deposition of intrinsic and doped Ge layers on $\mathrm{Si}(001)$ for microelectronics and optoelectronics purposes," Journal of Crystal Growth, vol. 274, no. 1-2, pp. 90-99, 2005.

[24] A. Nayfeh, C. O. Chui, K. C. Saraswat, and T. Yonehara, "Effects of hydrogen annealing on heteroepitaxial-Ge layers on Si: surface roughness and electrical quality," Applied Physics Letters, vol. 85, no. 14, pp. 2815-2817, 2004.

[25] Y. Liu, M. D. Deal, and J. D. Plummer, "High-quality single-crystal Ge on insulator by liquid-phase epitaxy on $\mathrm{Si}$ substrates," Applied Physics Letters, vol. 84, no. 14, pp. 25632565, 2004.

[26] G. Vanamu, A. K. Datyel, and S. H. Zaidi, "Epitaxial growth of high-quality Ge films on nanostructured silicon substrates," Applied Physics Letters, vol. 88, no. 20, Article ID 204104, 2006.

[27] T. A. Langdo, C. W. Leitz, M. T. Currie, E. A. Fitzgerald, A. Lochtefeld, and D. A. Antoniadis, "High quality Ge on Si by epitaxial necking," Applied Physics Letters, vol. 76, no. 25, pp. 3700-3702, 2000.

[28] H.-C. Luan, D. R. Lim, K. K. Lee et al., "High-quality Ge epilayers on Si with low threading-dislocation densities," Applied Physics Letters, vol. 75, no. 19, pp. 2909-2911, 1999.

[29] T. H. Loh, H. S. Nguyen, C. H. Tung et al., "Ultrathin low temperature SiGe buffer for the growth of high quality Ge epilayer on $\mathrm{Si}(100)$ by ultrahigh vacuum chemical vapor deposition," Applied Physics Letters, vol. 90, no. 9, Article ID 092108, 2007.

[30] Z. W. Zhou, C. Li, H. K. Lai, S. Y. Chen, and J. Z. Yu, "The influence of low-temperature Ge seed layer on growth of highquality Ge epilayer on $\mathrm{Si}(100)$ by ultrahigh vacuum chemical vapor deposition," Journal of Crystal Growth, vol. 310, no. 10, pp. 2508-2513, 2008. 
[31] C. G. Van de Walle, "Band lineups and deformation potentials in the model-solid theory," Physical Review B, vol. 39, no. 3, pp. 1871-1883, 1989.

[32] Y. H. Chen, C. Li, Z. W. Zhou et al., "Room temperature photoluminescence of tensile-strained $\mathrm{Ge} / \mathrm{Si}_{0.13} \mathrm{Ge}_{0.87}$ quantum wells grown on silicon-based germanium virtual substrate," Applied Physics Letters, vol. 94, no. 14, Article ID 141902, 2009.

[33] C. Li, Y. H. Chen, Z. W. Zhou, H. K. Lai, and S. Y. Chen, "Enhanced photoluminescence of strained Ge with a $\delta$-doping SiGe layer on silicon and silicon-on-insulator," Applied Physics Letters, vol. 95, no. 25, Article ID 251102, 2009.

[34] Y. H. Chen, C. Li, Z. W. Zhou, H. K. Lai, and S. Y. Chen, "Quantum-confined direct band transitions in tensile strained Ge/SiGe quantum wells on silicon substrates," Nanotechnology, vol. 21, no. 11, Article ID 115207, 2010.

[35] J. Wagner and L. Viña, "Radiative recombination in heavily doped p-type germanium," Physical Review B, vol. 30, no. 12, pp. 7030-7036, 1984. 


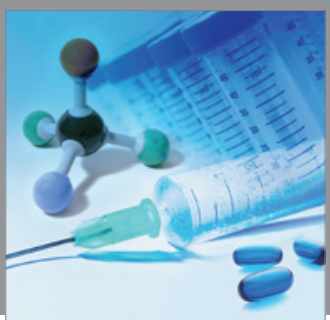

International Journal of

Medicinal Chemistry

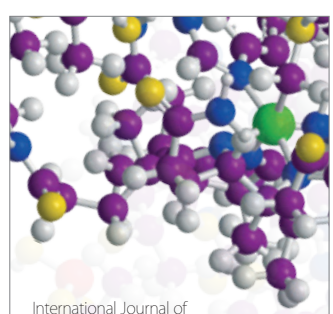

Carbohydrate Chemistry

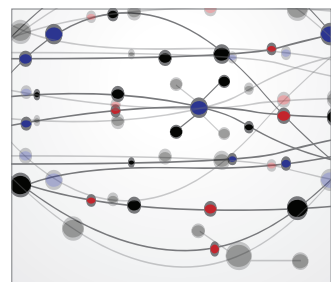

The Scientific World Journal
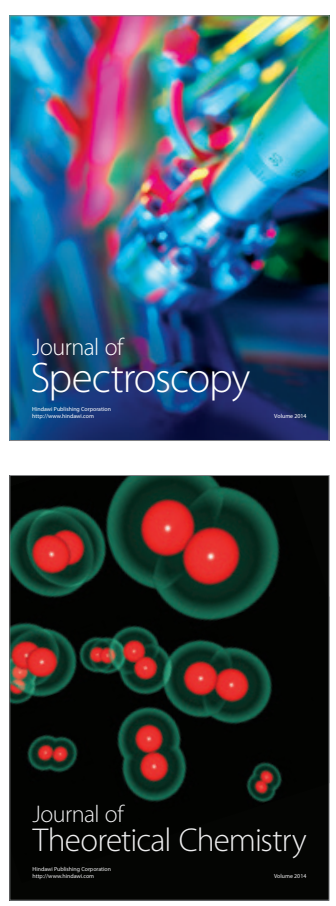
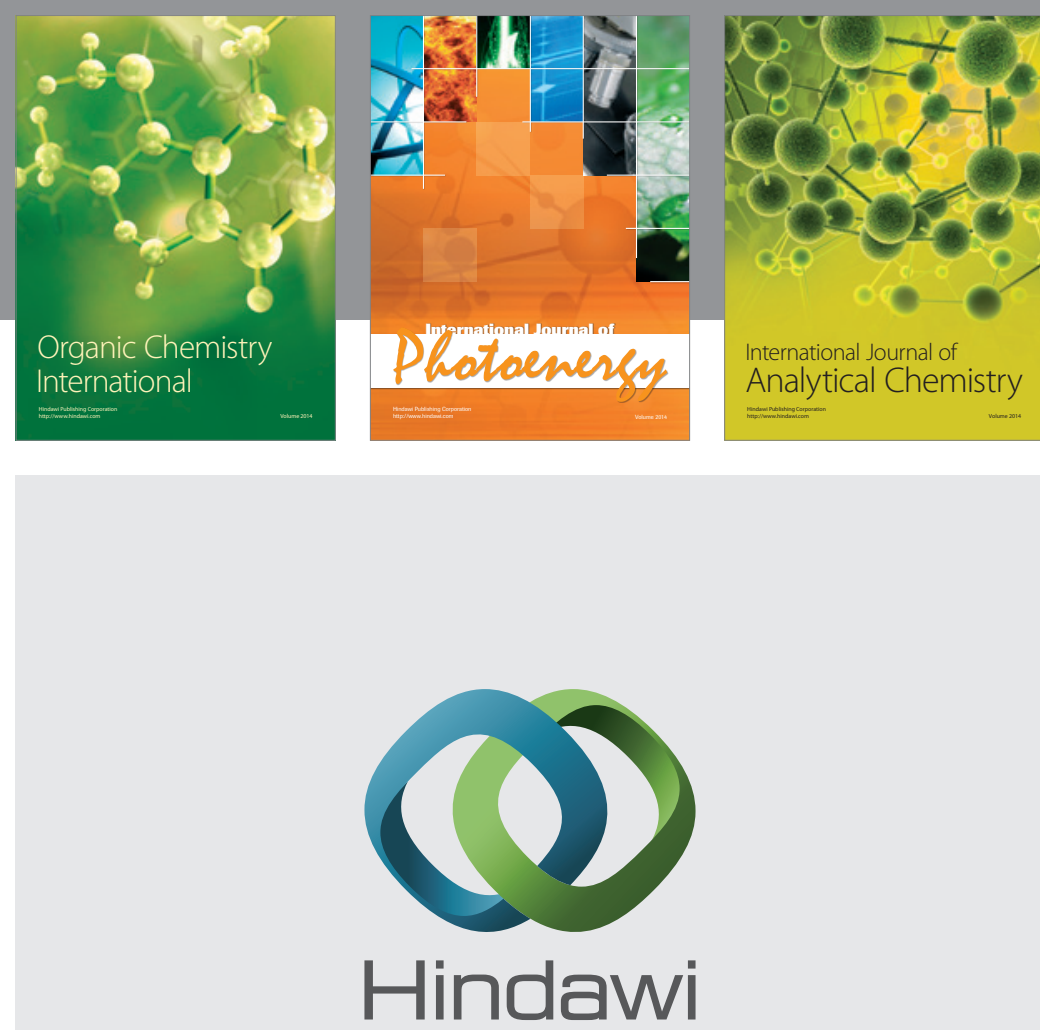

Submit your manuscripts at

http://www.hindawi.com
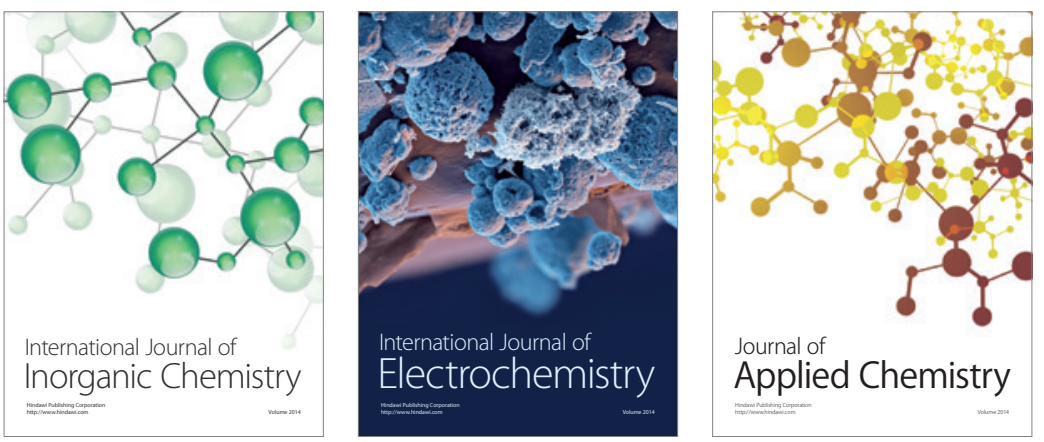

Journal of

Applied Chemistry
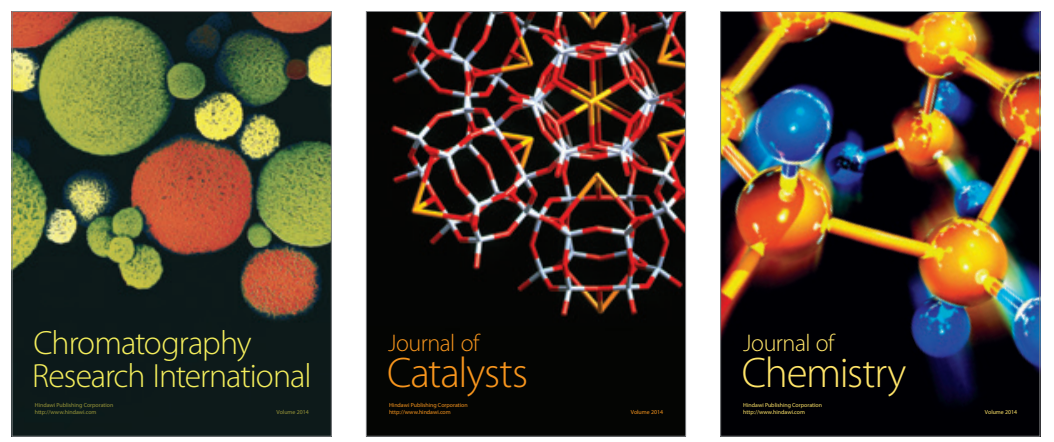
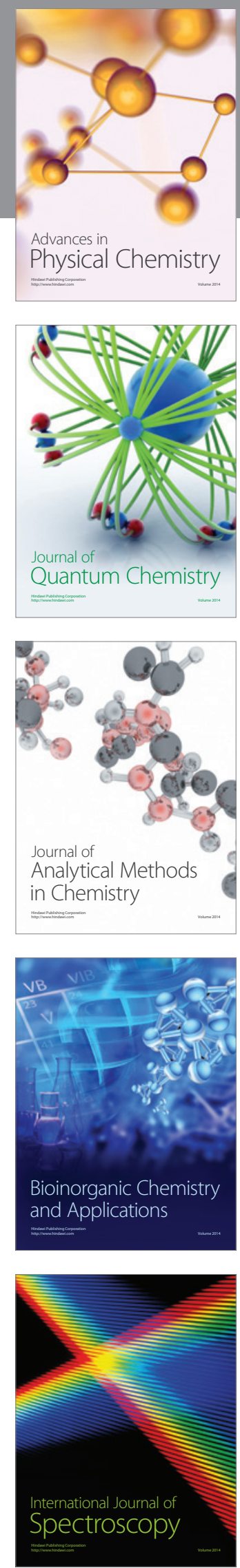